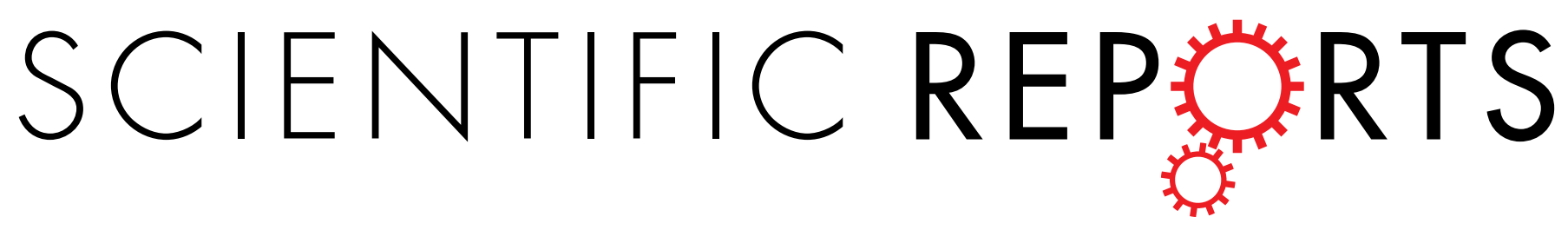

\title{
Erratum: Mirror neuron therapy for hemispatial neglect patients
}

Wei Wang, Xin Zhang, Xiangtong Ji, Qian Ye, Wenli Chen, Jun Ni, Guangyu Shen, Bing Zhang, Ti-Fei Yuan \& Chunlei Shan

Scientific Reports 5:8664; doi: 10.1038/srep08664; published online 02 March 2015; updated on 26 August 2015

In the original version of this Article, Wei Wang and Xin Zhang were incorrectly listed as being affiliated with 'Shanghai University of Traditional Chinese Medicine, Shanghai. 201203, China' (affiliation 5) and Chunlei Shan was listed as not being affiliated with 'Shanghai University of Traditional Chinese Medicine, Shanghai. 201203, China.' These errors have been corrected in the PDF and HTML versions of the Article. 